\title{
Dual targeting of Angiopoetin-2 and VEGF potentiates effective vascular normalisation without inducing empty basement membrane sleeves in xenograft tumours
}

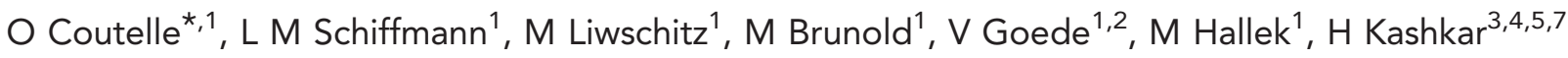
and U T Hacker ${ }^{1,6,7}$

${ }^{1}$ Department I for Internal Medicine, University of Cologne, Kerpener Strasse 62, 50924 Cologne, Germany; ${ }^{2}$ Deptartment for Geriatric Care, St Marien Hospital, Kunibertskloster 11-13, 50668 Cologne, Germany; ${ }^{3}$ Institute for Medical Microbiology, Immunology and Hygiene, Medical Faculty, University of Cologne, Goldenfelsstraße 19-21, 50935 Cologne, Germany; ${ }^{4}$ Cologne Excellence Cluster on Cellular Stress Responses in Aging-Associated Diseases (CECAD), Medical Faculty, University of Cologne, Robert-Koch-Straße 39, 50931 Cologne, Germany; ${ }^{5}$ Center for Molecular Medicine Cologne (CMMC), Robert-Koch-Straße 39, 50931 Cologne, Germany and ${ }^{6}$ University Cancer Center Leipzig, Liebigstraße 20, 04103 Leipzig, Germany

Background: Effective vascular normalisation following vascular endothelial growth factor (VEGF) inhibition is associated with endothelial cell regression leaving empty basement membrane sleeves (BMS). These long-lived BMS permit the rapid regrowth of tumour vasculature upon treatment cessation and promote resistance to VEGF-targeting drugs. Previous attempts at removing BMS have failed. Angiopoietin-2 (Ang2) is a vascular destabilizing factor that antagonises normalisation. We hypothesised that Ang2 inhibition could permit vascular normalisation at significantly reduced doses of VEGF inhibition, avoiding excessive vessel regression and the formation of empty BMS.

Methods: Mice xenografted with human colorectal cancer cells (LS174T) were treated with low $\left(0.5 \mathrm{mg} \mathrm{kg}^{-1}\right)$ or high $\left(5 \mathrm{mg} \mathrm{kg}^{-1}\right)$ doses of the VEGF-targeting antibody bevacizumab with or without an Ang2 blocking peptibody L1-10. Tumour growth, BMS formation and normalisation parameters were examined including vessel density, pericyte coverage, adherence junctions, leakiness, perfusion, hypoxia and proliferation.

Results: Dual targeting of VEGF and Ang2 achieved effective normalisation at only one-tenth of the dose required with bevacizumab alone. Pericyte coverage, vascular integrity, adherence junctions and perfusion as prerequisites for improved access of chemotherapy were improved without inducing empty BMS that facilitate rapid vascular regrowth.

Conclusions: Dual targeting of VEGF and Ang2 can potentiate the effectiveness of VEGF inhibitors and avoid the formation of empty BMS.

Blood vessels are indispensible for tumour growth and metastasis. Therefore, anti-angiogenic drugs in particular those focusing on blocking the vascular endothelial growth factor (VEGF) pathway are part of standard therapy for diverse tumour entities such as colorectal, renal cell, hepatocellular and non-small cell lung cancer (Jain et al, 2006; Ellis and Hicklin, 2008). The clinical benefit of VEGF-targeting drugs besides inducing vascular regression results from their capacity to normalise the phenotype and function of the

\footnotetext{
*Correspondence: Dr O Coutelle; E-mail: oliver.coutelle@gmail.com

${ }^{7}$ These authors share senior authorship.

revised 23 November 2014; accepted 28 November 2014; published online 6 January 2015

(c) 2015 Cancer Research UK. All rights reserved 0007-0920/15
} 
surviving tumour blood vessels (Hurwitz et al, 2004; Sandler et al, 2006). Vascular normalisation has been shown to improve the penetration of chemo- and immunotherapy (Tong et al, 2004; Netti et al, 1999), convert the immunosuppressive microenvironment of tumours into an immunostimulatory one (Huang et al, 2012) and decrease intravasation of cancer cells (Mazzone et al, 2009; Rolny et al, 2011).

A major challenge of anti-angiogenic therapy therefore lies in devising strategies that improve vascular normalisation, extend its duration, and prevent the development of resistance to these interventions (reviewed in Bergers and Hanahan, 2008). Although, greater effectiveness of VEGF inhibition in terms of vascular regression might help to starve the tumour of oxygen and nutrients, excessive vessel pruning may occur at the cost of adequate delivery of chemotherapy, shifting the net balance towards hypoxia-driven rebound angiogenesis, particularly if the tumour vasculature retains the capacity to remodel its blood vessels. This escape phenomenon appears to be aided by durable scaffolds of vascular basement membrane and pericytes that persist after VEGF targeting (Inai et al, 2004; Mancuso et al, 2006). Therefore, strategies focusing not simply on increased vascular regression but on extending the normalisation window and preventing vascular regrowth are most likely to improve patient outcome. In pursuing this goal, treatments combining VEGFtargeting drugs with other anti-angiogenic agents are currently being developed (reviewed in Gerald et al, 2013).

Angiopoietin-2 (Ang2) is synthesised and secreted primarily by endothelial cells at sites of vascular remodelling, in response to VEGF or hypoxia (Fiedler et al, 2004). It interrupts Ang1-mediated vessel normalisation, resulting in impaired pericyte coverage, vessel destabilisation and increased vascular permeability, conditions conducive for sprouting angiogenesis. Indeed, it has recently been shown that Ang2 can directly induce sprouting angiogenesis by engaging integrin $\alpha \mathrm{v} \beta 5$ receptors (Felcht et al, 2012). In patients with metastatic colorectal cancer, we have previously shown that high serum Ang2 levels are associated with adverse response rate and clinical outcome to therapy with the VEGF-targeting antibody bevacizumab (Goede et al, 2010) and similar observations have been made in glioblastoma patients (Batchelor et al, 2010). Since Ang2 is a vascular destabilizing factor that antagonises vascular normalisation (Chae et al, 2010), we hypothesised that blocking Ang2 could have synergistic effects with VEGF inhibition especially in terms of vascular normalisation. Dual inhibition of VEGF and Ang2 has already been demonstrated to have additive inhibitory effects on slowing tumour growth in a murine xenograft model by limiting sprouting angiogenesis, and increasing apoptosis (Hashizume et al, 2010). Most recently, CrossMab, a cross-specific antibody targeting VEGF-A and Ang2 has been shown to reduce metastasis in an experimental tumour model (Kienast et al, 2013). However, effects of Ang2 inhibition on vascular normalisation and in particular its impact on BMS under conditions of submaximal VEGF inhibition have not yet been studied. Here, we investigated whether inhibition of Ang2 with a recombinant peptide-Fc fusion protein (peptibody) that selectively inhibits Ang2 binding to its tyrosine kinase receptor (Tie2) could potentiate the efficacy of reduced doses of the humanised VEGF-targeting antibody bevacizumab on vascular normalisation and whether under these conditions the formation of BMS could be limited in a murine xenograft tumour model of colorectal cancer.

\section{MATERIALS AND METHODS}

Cell lines, cell culture. The human colon carcinoma cell line LS174T was purchased from ATCC (Bethesda, MD, USA) and maintained in RPMI-1640 supplemented with $10 \%$ fetal calf serum,
$2 \mathrm{mM}$ L-glutamine, $100 \mu \mathrm{g} \mathrm{ml}^{-1}$ streptomycin and $100 \mathrm{U} \mathrm{ml}^{-1}$ penicillin (Biochrom, Berlin, Germany).

Animal tumour models. Balb/cA nude mice were purchased from Charles River (Sulzfeld, Germany). Xenograft tumours were generated by injecting human colorectal cancer cells LS174T $\left(3 \times 10^{6}\right)$ subcutaneously into the flank region of 6- to 8-week-old mice. From the time of palpable tumour development, animals were split into treatment groups of six animals each and received twice weekly intraperitoneal injections of anti-human VEGF antibody bevacizumab at either $5 \mathrm{mg} \mathrm{kg}^{-1}$ (B100) or at $0.5 \mathrm{mg} \mathrm{kg}^{-1}$ body weight (B10), the Ang2 neutralizing Fc-fusion protein L1-10 ('L1') (Amgen Inc., Cambridge, MA, USA) at $4 \mathrm{mg} \mathrm{kg}^{-1}$ thrice weekly or combinations thereof L1/B100 or L1/B10. Tumour size was measured every other day using precision calipers. Tumour volume was calculated as length $\times$ width $^{2} \times \pi 6$ and expressed as average \pm s.e.m.

Ethics statement. Animals were housed in the animal care facility of the University of Cologne under standard pathogen-free conditions with a 12-h light/dark schedule and provided with food and water ad libitum. Ethical approval was obtained with explicit permission of the Landesamt für Natur, Umwelt \& Verbraucherschutz (LANUV) of the state Northrhine Westphalia, Leibnizstraße 10, 45659 Recklinghausen, Germany. All animal experiments were performed under permit number 9.93.2.10.31.07.141.

Histology and microscopy. Tumour tissue samples were snapfrozen in O.C.T. for storage and further processing. Fresh cryosections $(20 \mu \mathrm{m})$ were prepared and fixed in $3 \%$ paraformaldehyde for $10 \mathrm{~min}$, followed by incubation in blocking solution (10\% normal goat serum, Invitrogen, Karlsruhe, Germany) and incubation with appropriate primary and secondary antibodies. Cryosections were mounted in Mowiol mounting medium (Calbiochem, Schwalbach, Germany) and examined with a motorised inverted microscope (Olympus IX81 or IX71 equipped with Cell $\wedge \mathrm{R}$ Imaging Software, Tokyo, Japan) using a $\times 60 / 1.45$ numerical aperture Planapo oil objective.

Immunohistochemistry. Endothelial cells were stained with rat monoclonal anti-mouse CD31 antibody (PEACAM-1; clone MEC 13.3, 1:500; BD Pharmingen, Heidelberg, Germany) and detected using an Alexa Fluor 488-conjugated polyclonal goat anti-rat antibody (1:500, Invitrogen). Pericytes were stained with Cy3conjugated mouse monoclonal anti- $\alpha$-smooth muscle actin antibody (1:1000, $\alpha$ SMA clone 1 A4, Sigma-Aldrich, Munich, Germany). VE-Cadherin adherence junctions were stained with a rat anti-mouse VE Cadherin antibody $(1: 400$, Clone 11D4.1, BD Pharmingen). Vascular basement membrane was identified with rabbit polyclonal anti-type IV collagen antibody (1:100; ab6586, Abcam, Cambridge, UK). Tumour cell proliferation was assessed with a rabbit Ki67 polyclonal antibody $(1: 200$, Menarini Diagnostics, Berlin, Germany).

Vascular leakiness, hypoxia and perfusion studies. Vessel leakiness was assessed by i.v. injection of $100 \mu \mathrm{l}$ of FITC-dextran (15 mg ml $\mathrm{mg}^{-1}$ in PBS, MW 2000 000; Sigma-Aldrich) into the tail vein of tumour bearing mice $30 \mathrm{~min}$ before animals were killed. For hypoxia studies, $200 \mu \mathrm{l}$ of pimonidazole hydrochloride (15 $\mathrm{mg} \mathrm{ml}^{-1}$, ArtimmunAnalytik, Kelkheim, Germany) was injected into the tail vein of tumour bearing mice $30 \mathrm{~min}$ before animals were killed by cervical dislocation. The formation of pimonidazole adducts in hypoxic tumour areas was detected with a FITC monoclonal anti-pimonidazole antibody (1:50, clone 4.3.11.3, ArtimmunAnalytik). To assess the effect of drug treatment on tumour vessel perfusion, $200 \mu \mathrm{l}$ of $15 \mathrm{mg} \mathrm{kg}^{-1}$ Hoechst-33342 (Sigma-Aldrich) was injected into the tail vein and tissues samples were collected and processed for cryosectioning after $3 \mathrm{~min}$. All tumours were excised and snap frozen in O.C.T for storage and further processing. 
Image acquisition and analysis. The fractional area of CD31 represents the percentage of $\mathrm{CD} 31$ immunoreactivity in viable tumour tissue typically examined in 100x images of tumour sections using the ImageJ software (http://rsbweb.nih.gov/ij/) on 5-10 microscopic images per tumour with an empirically determined threshold of 40 . At least three tumours were analysed from vascular hot spots in four quadrants of the tumour and in the centre. Pericyte coverage was quantified from 10 to $12-\mu \mathrm{m}$-thick $\mathrm{z}$-stacks of individual linear vessel stretches with a diameter of at least $6 \mu \mathrm{m}$ in tumour vascular hotspots at $600 \mathrm{x}$ magnification from sections stained for CD31 and $\alpha$ SMA. At least five vessels were analysed from 3 to 4 tumours per group. The endothelial cell outline was marked with the freehand selection tool in ImageJ and the area density of $\alpha \mathrm{SMA}$ in this region was calculated using an empirically determined threshold value of 20-25. VE-cadherin density was determined in 10 - to $12-\mu \mathrm{m}$-thick $\mathrm{z}$-stacks of $600 \mathrm{x}$ fluorescence microscopic images of individual vessels, stained for the endothelial adherence junction protein VE-cadherin. To determine the amount of immunoreactivity of VE-Cadherin the endothelium was outlined with the freehand selection tool in Image J and the area density of VE-Cadherin in the outlined region was calculated using the autoset threshold function of ImageJ. Vessel leakiness was analysed in 100x images of tumours of FITCdextran-injected mice and the percentage of leaky vessels (identified by dextran extravasation into the surrounding tumour tissue) was calculated among perfused vessels (FITC-Dextran and CD31 positive). Non-perfused vessels were not included. At least four tumours per group and 5-9 images were analysed, depending on tumour size. Tumour vessel perfusion was analysed in $400 \mathrm{x}$ Multiple-Alignment-Images (Cell^ $\mathrm{R}$ Imaging Software, Olympus, Munich, Germany) of whole tumour sections. In all, 3-7 tumours per group were analysed. Perfusion was quantified by calculating the fraction of Hoechst-33342 dye positive tumour area per area density of CD31 to account for the differences in vessel density using the threshold values automatically determined by ImageJ. The perfusion distance represents the distance to half maximum signal intensity and was determined by measuring the concentration gradient of Hoechst-33342 from the vessel lumen into the tumour tissue along radial lines of 50-100 pixel width drawn from the vessel lumen along the Hoechst-33342 concentration gradient of non-confluent areas of Hoechst-33342 extravasation. The plotprofile function of ImageJ was used to generate a florescence intensity profile chart. Three measurements from 10 vessels per tumour were taken. At least three tumours per group were analysed. The intensity values were expressed as percentage and plotted in line charts as a function of distance from the blood vessel wall. The hypoxic tumour fraction was calculated as the percentage of pimonidazole immunoreactivity per whole tumour section, using ImageJ. Non-viable/necrotic tumour areas were identified visually as lacking the proliferation marker Ki67 and showing nonspecific or absent staining for 4',6-Diamidin-2-phenylindol (DAPI) and manually outlined for quantification using Adobe Photoshop. Basement membrane sleeves were estimated by calculating the area density of type IV collagen immunoreactivity per CD31 area density using ImageJ with an empirically determined threshold value of 40 .

Statistical analysis. All results were expressed as mean \pm s.e. Differences between experimental groups were analysed by unpaired Student's t test. $P<0.05$ was considered as statistically significant.

\section{RESULTS}

Inhibition of Ang-2 was studied in the presence of high, intermediate and low VEGF levels, in a xenograft tumour model of VEGF expressing human colorectal cancer cells (LS174T). In these xenografted mice circulating human VEGF levels were left either unopposed (Ctrl) or subject to inhibition by the VEGF antibody bevacizumab at $5 \mathrm{mg} \mathrm{kg}^{-1}$ (B100) or $0.5 \mathrm{mg} \mathrm{kg}^{-1}$ (B10) twice weekly. A bevacizumab concentration of $0.5 \mathrm{mg} \mathrm{kg}^{-1}$ (B10) was chosen because it reduced the serum VEGF level and tumour growth rate to an intermediate level between the typical $5 \mathrm{mg} \mathrm{kg}^{-1}$ dose (B100) and the controls (Supplementary Figure S1A and B). At higher bevacizumab doses of $1.5 \mathrm{mg} \mathrm{kg}^{-1}$ (B30), tumour growth was no longer significantly different from the B100-treated cohort (Supplementary Figure S1B). In additional cohorts, circulating Ang2 was inhibited by the peptibody L1 (Amgen) either alone (L1) or in combination with high and intermediate levels of VEGF inhibition (L1/B100 and L1/B10).

Tumour growth following either low-dose VEGF inhibition (B10) or inhibition with the Ang2 inhibitor L1 was reduced to $50 \%$ of the untreated controls. Remarkably, when B10 was combined with Ang2 inhibition (L1/B10) the tumour growth was further reduced to just one-quarter of the controls. A comparable level of growth inhibition with VEGF inhibition alone was achievable only by increasing the dose of bevacizumab 10-fold (B100). Dual inhibition with L1/B100, however, showed no additional benefit (Figure 1A).

The tumour growth rates correlated broadly with the reduction in blood vessels as represented by the fraction of CD31-positive area and consistent with the anti-angiogenic effects of VEGF and Ang2 inhibition (Figure 1B). Whereas L1 and B10 treatment reduced the vessel density by a third compared with the untreated controls, dual inhibition with L1/B10 produced a reduction to $50 \%$ of controls. The vessel density in the B100- and L1/B100-treated animals was even lower at just $30 \%$ of the controls (Figure 1B). Together, these findings indicate that low-dose VEGF inhibition may be augmented by simultaneous inhibition of Ang2 in terms of tumour growth inhibition and vessel density.

Angiopoetin-2 is thought to interfere with the recruitment of pericytes and smooth muscle cells that are required for the stabilisation of maturing blood vessels. To examine the pericyte coverage, we chose $\alpha \mathrm{SMA}$ as a marker of mature pericytes over earlier markers such as NG2 and PDGFR- $\beta$ because we were mostly interested in the later stages of tumour development after 18 days of treatment (Figure 2A). In the control tumours, pericytes identified by $\alpha \mathrm{SMA}$ immunoreactivity were abundant, but were only loosely associated with tumour vessels reaching $54 \%$ coverage in agreement with the previous measurements (Dang et al, 2008). Compared with controls, there was no significant change in $\alpha \mathrm{SMA}$ pericyte coverage in response to VEGF inhibition alone, however, the pericyte coverage was significantly increased in all of the groups subjected to Ang2 inhibition. Treatment with L1, L1/B10 and L1/B100 increased the $\alpha \mathrm{SMA}$ pericyte coverage to $68 \%, 78 \%$ and $69 \%$, respectively (Figure $2 \mathrm{~A}$ ). In addition, $\alpha \mathrm{SMA}$ pericytes appeared to be more orderly attached to the tumour vessels with fewer partially attached pericytes. A separate analysis of the area density of NG2 per CD31 area produced broadly similar results, suggesting that the pericyte area per vessel area is increased upon dual inhibition rather than a shift in the pericyte phenotype occurring (Supplementary Figure S2).

Endothelial permeability is regulated in part by VE-cadherin adherence junctions. Although there was a trend to more linear and dense VE-cadherin staining in response to VEGF treatment alone, a statistically significant increase was observed only in the groups receiving Ang2 inhibition either alone or in combination with VEGF inhibition (Figure 2B). By comparison, the distribution of VE-cadherin in the blood vessels of control tumours was disorganised and of weak intensity. This contrasted with the more organised and regular appearance observed in all tumours subjected to Ang2 inhibition including L1, L1/B10 and L1/B100 (Figure $2 \mathrm{~B}$ ). To functionally assess changes in barrier function, we evaluated the permeability of the tumour vessels by injecting 

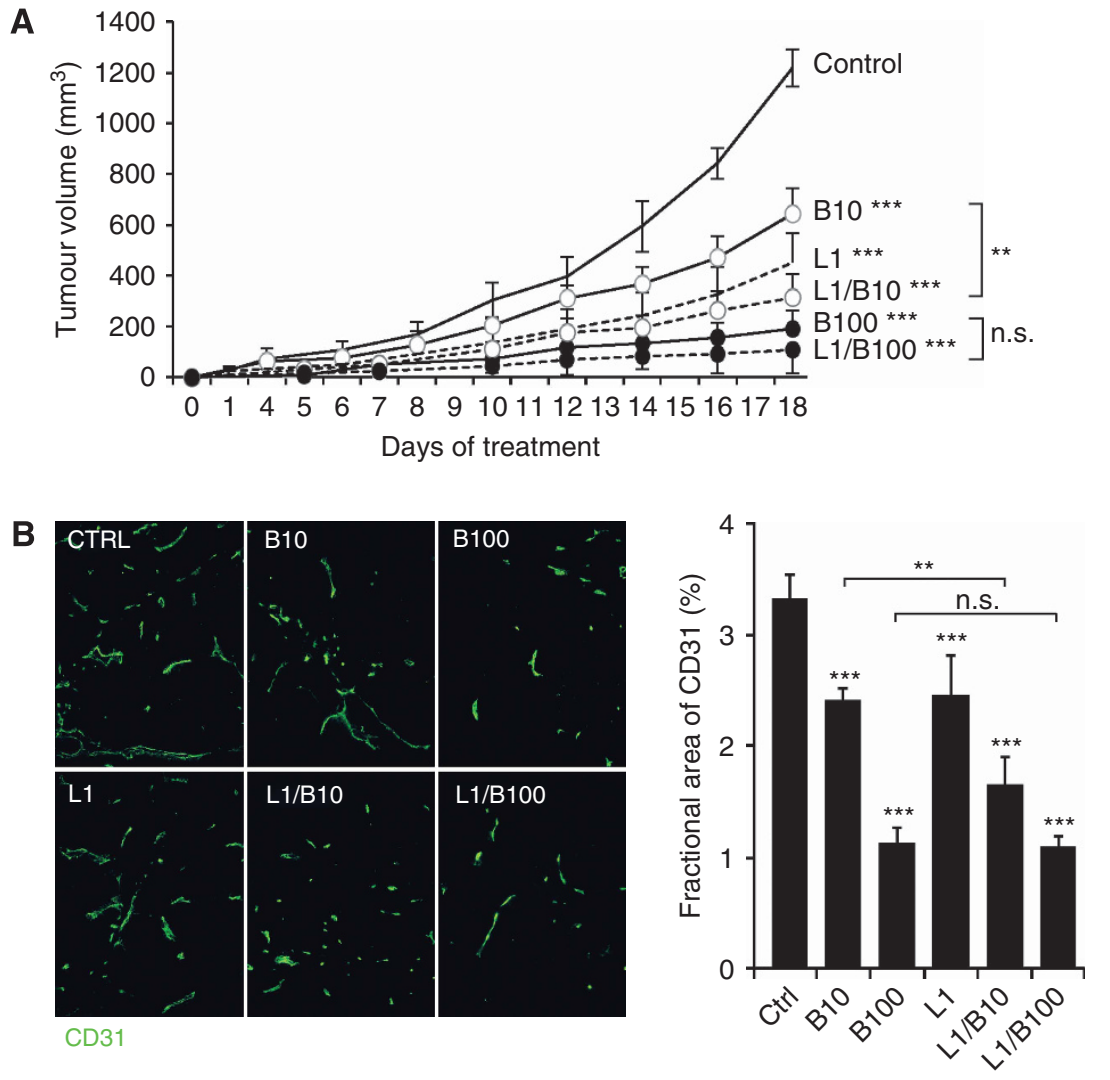

Figure 1. Xenograft tumour growth and vascularity in response to VEGF and Ang2 inhibition (A) Tumour growth of LS174T xenografts in mice treated with full dose $5 \mathrm{mg} \mathrm{kg}^{-1}$ (B100) or low dose $0.5 \mathrm{mg} \mathrm{kg}^{-1}$ (B10) bevacizumab or the Ang2 inhibitor (L1) and combinations thereof (L1/B10 and L1/B100). (B) Fractional area of CD31 immunoreactivity corresponding to endothelial cells in histological tumour sections cut through the centre of each tumour at the end of the treatment period. Overall, the reduction in vessel area density in the treatment groups mirrors their growth kinetics. Symbols above individual bars indicate statistical significance vs the control group. Symbols above brackets indicate the statistical significance between these two groups. For a comprehensive statistical comparison of the treatment groups, please refer to Supplementary Table 1. ${ }^{\star \star \star} P \leq 0.001,{ }^{\star \star} P \leq 0.01$.

fluorescent high molecular weight dextran into the tail vein of tumour bearing mice (Figure 3A). In all, 66\% of the vessels in control tumours showed significant extravasation of dextran into the interstitial space. In comparison, the proportion of leaky tumour vessels was already significantly lower in B10- and B100treated tumours with $45 \%$ and $33 \%$ leaky vessel, respectively, and $40 \%$ upon L1 treatment alone. However, the most striking improvement in barrier function was achieved by dual inhibition with just $22 \%$ leaky vessels in L1/B10 and 21\% in L1/B100 tumours. Together, these findings support the view that Ang2 inhibition enhances vascular normalisation parameters in concert with VEGF inhibition.

Given these improvements in barrier function upon dual targeting of VEGF and Ang2, we expected the interstitial fluid pressure to be reduced leading to enhanced perfusion with therapeutic drugs. The agent of choice in the context of colorectal cancer, would be 5-fluorouracil. However, fluoropyrimidines have themselves been shown to reduce tumour growth by disrupting the tumour vasculature in murine xenograft models (Ooyama et al, 2008). We therefore choose to use Hoechst-33342 as an established surrogate for chemotherapy who's uptake has been shown to parallel that of a number of cytotoxic drugs and to correlate with cellular sensitivity to these drugs (Lalande et al, 1981). The fluorescent stain Hoechst-33342 (H33342) was intravenously injected into tumour bearing mice. The ratio of $\mathrm{H} 33342$-stained tumour area and CD31 area was calculated to assess the area of H33342 perfusion (Figure 3B). The perfusion area compared with untreated controls increased 2.6-fold for the B100 group, 2-fold for tumours treated with $\mathrm{L} 1$ alone and more than 3-fold for the combination groups L1/B10 and L1/100 (Figure 3B). To complement this assessment, we also compared the concentration gradients of $\mathrm{H} 33342$ from the vessel lumen into the tumour tissue and found that dual inhibition (L1/B10 and L1/B100) increased the penetration depth, that is, the distance to half maximal signal intensity by more than $50 \%$ from $36 \mu \mathrm{m}$ in the controls to 56 and $54 \mu \mathrm{m}$, respectively (Figure 3C). Remarkably, VEGF or Ang2 inhibition alone had no such effect. While the observed increase in penetration depth suggested improved vascular normalisation in areas of adequate blood supply, other tumour areas experience vascular pruning and regression as part of the normalisation process and the overall impact of these changes on tumour cell viability, hypoxia and death remains unclear.

To address this issue, we stained hypoxic tumour areas with pimonidazole and proliferating tumour areas with the proliferation marker Ki67 (Figure 4A). Control tumours showed large areas of proliferation (red) with little hypoxia (green) or non-viable/necrotic tissue identified as Ki67, pimonidazole and DAPI negative. The hypoxic tumour fraction increased significantly following VEGF inhibition but this was accompanied by only a moderate increase in cell death. In contrast, significantly more cell death but less hypoxia was observed upon Ang2 inhibition consistent with the early dependence of tumour angiogenesis on Ang2 (Nasarre et al, 2009). Note also that the sum of both the hypoxic and the necrotic tumour fraction was greatest in the dual inhibition groups that also showed the least tumour growth (Figure 1A). Upon dual inhibition, the hypoxic tumour area decreased further, while the 
A
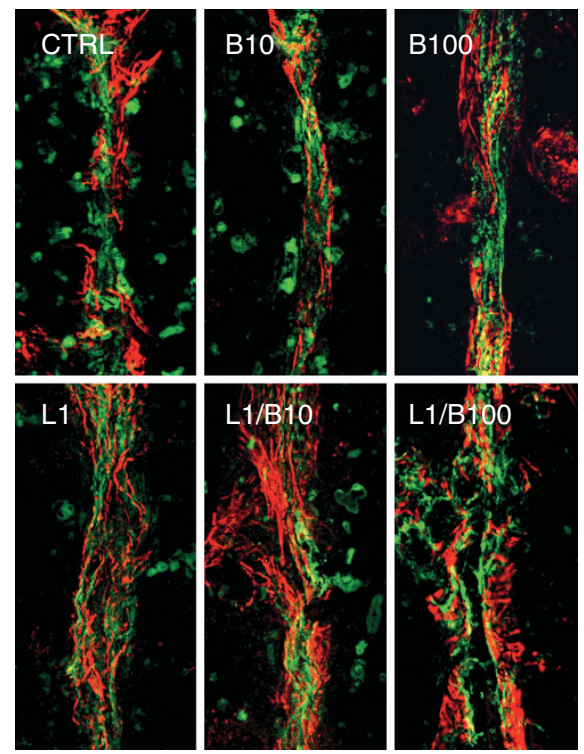

aSMA CD31

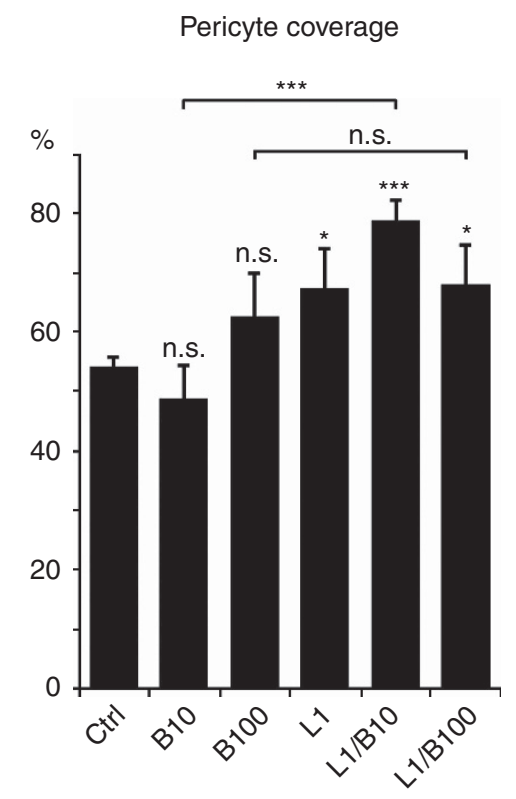

B
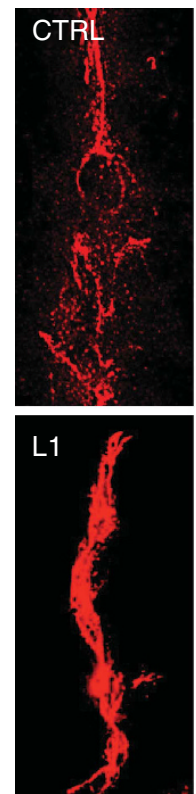

VE-cadherin
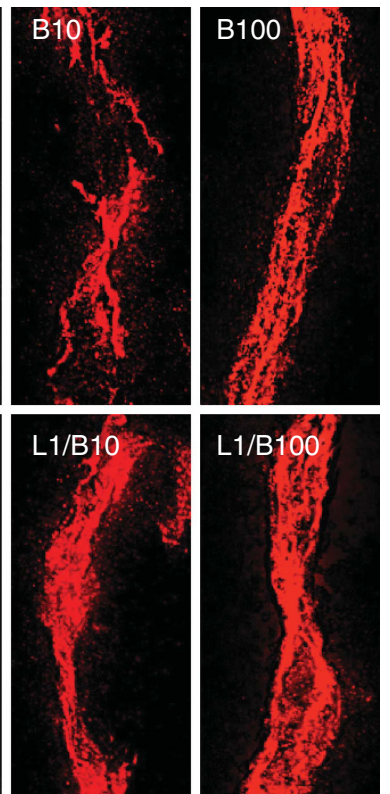

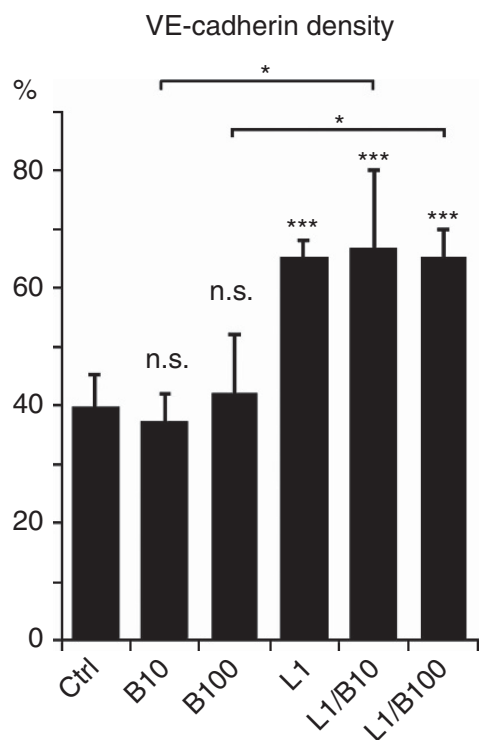

Figure 2. Treatment-related changes in pericyte coverage and VE-cadherin adherence junctions. (A) Representative semiconfocal immunofluorescence microscopy images of tumour blood vessels (CD31, green) and pericytes ( $\alpha$ SMA, red) and quantification of pericyte coverage. Note the significant increase in pericyte coverage in the groups upon Ang2 inhibition and in particular in the dual inhibition groups. (B) VE-cadherin expression in representative images of tumour vessels and quantification of area density of VE-cadherin staining. Although there is a trend to more linear and dense VE-cadherin staining in response to VEGF treatment alone, a significant increase is observed only in the group upon Ang2 inhibition and in the dual inhibition groups. Analyses after 18 days of treatment. Symbols above individual bars indicate statistical significance vs the control group. Symbols above brackets indicate the statistical significance between these two groups. For a comprehensive statistical comparison of the treatment groups, please refer to Supplementary Table 1. ${ }^{\star} P \leq 0.05,{ }^{\star \star \star} P \leq 0.001$.

non-viable/necrotic fraction increased (L1/B10 and L1/B100) suggesting that cell death occurred at the expense of the hypoxic tumour fraction. Given that Ang2 is required for vascular remodelling in response to hypoxia and other proangiogenic stimuli, we evaluated treatment-related changes in the remodelling capacity of the tumour vasculature by examining basement membrane deposits. Basement membranes are deposited in the process of new blood vessel formation and persist even after endothelial cells regress, leaving a record of previous angiogenic activity (Inai et al, 2004). We therefore assessed the amount of basement membrane deposition by collagen IV staining (Figure 4B). In the bevacizumab-treated groups, collagen IV staining showed a dense network of BMS (collagen IV deposits lacking associated endothelial cells) consistent with massive tumour vessel turnover. In contrast, BMS were sparse in the presence of Ang2 inhibition, indicating that the angiogenic remodelling activity in the presence of Ang2 inhibition was suppressed and the vascular plasticity window was closed (Figure 4B). 

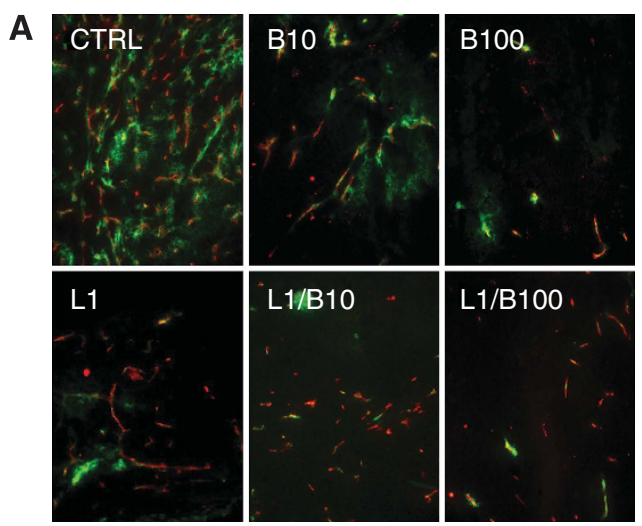

CD31 FITC-dextran

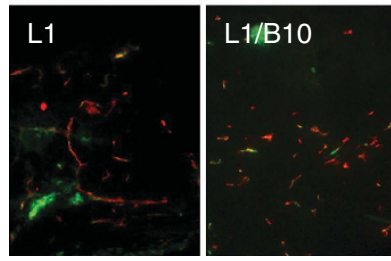

B
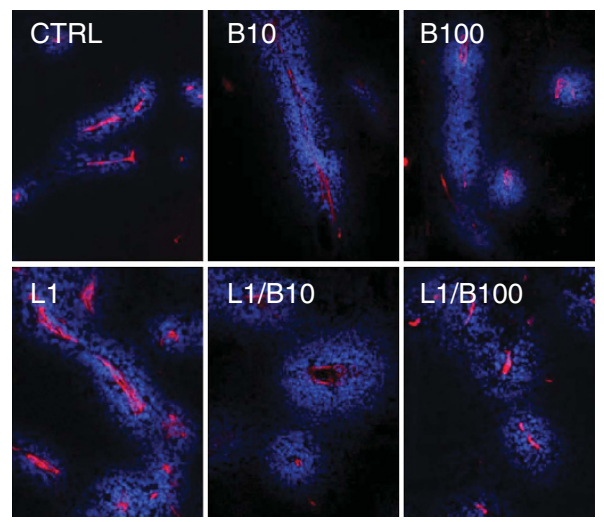

CD31 Hoechst 33342

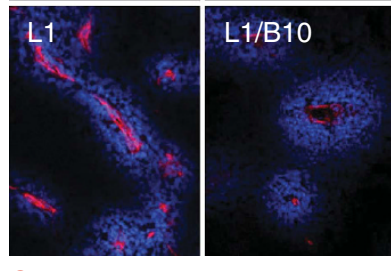

C

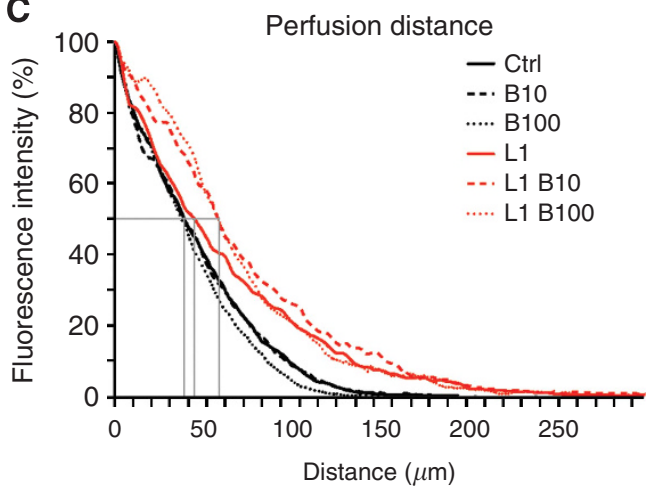

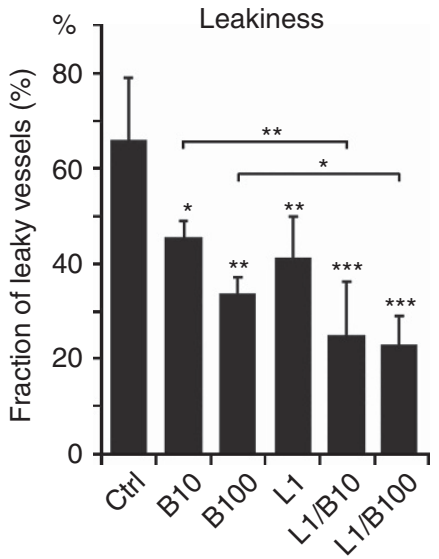
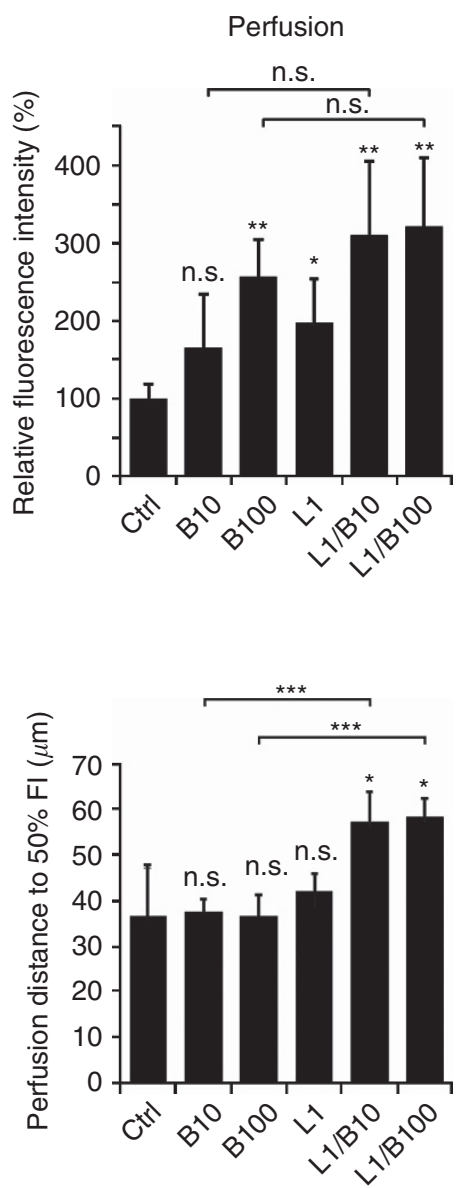

Figure 3. Treatment-related changes in endothelial barrier function and tumour perfusion. (A) Tumour vessels (CD31, red) perfused with or leaking FITC-dextran (green) 30 min after tail-vein injection detected in cryosections of LS174T tumours and quantification of the fraction of leaky blood vessels in each treatment group. (B) Perfusion of LS174T tumours with Hoechst-33342 dye (blue) 3 min after tail-vein injection. Blood vessels were stained with CD31 (red). Quantification of the area density of Hoechst-33342 per area density of CD31 based on multiple alignment images. There was a trend towards improved perfusion upon addition of $\mathrm{L} 1$ to B10 or B100 but this did not reach statistical significance. (C) The perfusion distance represents the radial distance of Hoechst-33342 signal intensity along the gradient measured from the vessel lumen into the peripheral tumour tissue and quantification of the perfusion distance until the half maximal intensity was reached. In the dual inhibition groups (L1/B10 and L1/B100), the perfusion distance is $>50 \%$ greater than in controls or tumours or tumours treated with either Ang2 or VEGF inhibition alone. Symbols above individual bars indicate statistical significance vs the control group. Symbols above brackets indicate the statistical significance between these two groups. For a comprehensive statistical comparison of the treatment groups, please refer to Supplementary Table 1. ${ }^{*} \leq \leq 0.05$, $\star \star P \leq 0.01, * \star * P \leq 0.001$. 
A

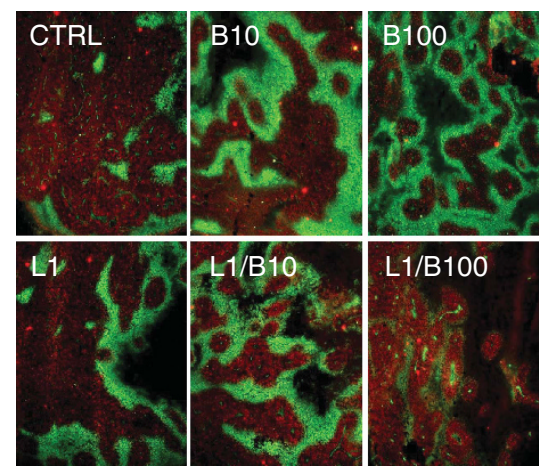

Ki67 pimonidazol

\section{B}
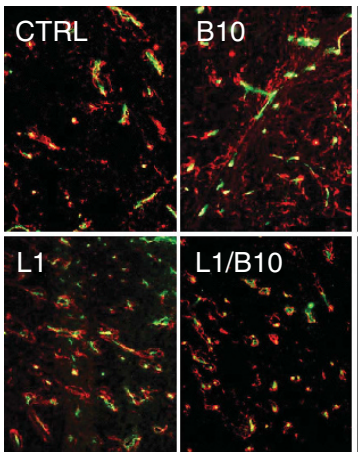

Collagen IV CD31

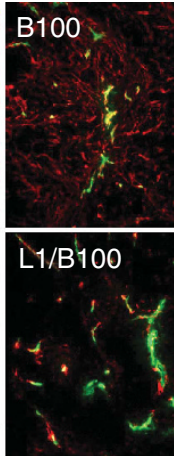

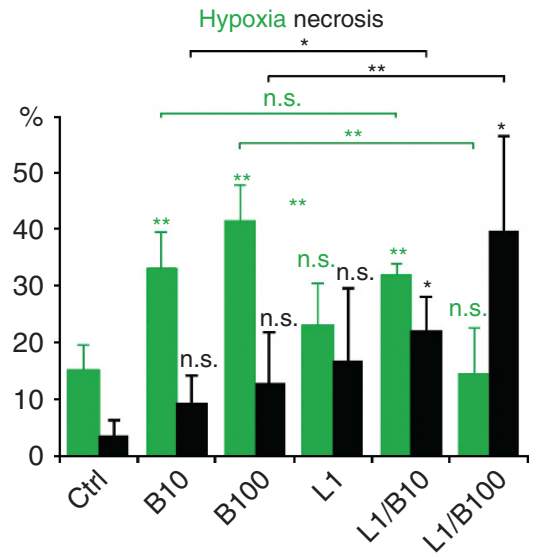

BM sleeves

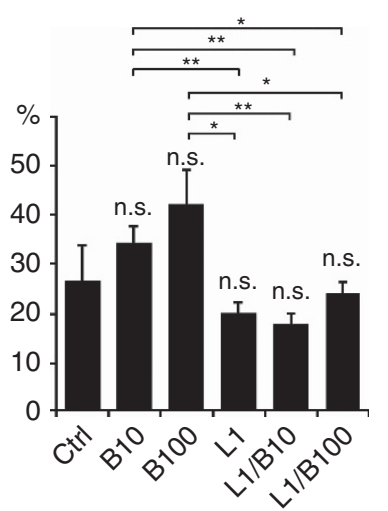

Figure 4. Treatment-related changes in tumour hypoxia, viability and basement membrane sleeves. (A) Immunohistochemistry and quantification of areas of hypoxia marked by the formation of pimonidazole adducts (green) and tumour cell proliferation identified by Ki67 staining (red). Nonviable tumour areas were identified and quantified as lacking pimonidazole, Ki67 and DAPI (not shown) staining. Note that hypoxia increases in all of the treatment groups except for L1/B100, where the proportion of cell death is increased at the expense of hypoxic tumour tissue. (B)

Immunohistochemistry of basement membrane deposits (type IV-collagen, red) and vascular endothelial cells (CD31, green) in tumour sections and quantification of the excess of basement membrane (BM) deposits in relation to endothelial cells ('sleeves'). VEGF inhibition is associated with increased BM deposition and vascular regression leading to empty BM sleeves. This is significantly reduced upon Ang2 inhibition. Symbols above individual bars indicate statistical significance vs the control group. Symbols above brackets indicate the statistical significance between these two groups. For a comprehensive statistical comparison of the treatment groups, please refer to Supplementary Table $1 .{ }^{\star} P \leq 0.05,{ }^{\star \star} P \leq 0.01$.

\section{DISCUSSION}

Dual inhibition of VEGF and Ang2 has previously been shown to synergistically inhibit sprouting angiogenesis leading to reduced proliferation and increased apoptosis of tumour cells (Hashizume et al, 2010). In the clinical setting, however, the addition of antiVEGF therapy to systemic chemotherapy has often improved patient outcome when compared with chemotherapy alone (Hurwitz et al, 2004; Sandler et al, 2006), suggesting that anti-angiogenic therapy improved access of chemotherapy by a mechanism described as vascular normalisation (Jain, 2001). The relative contribution of vascular pruning versus normalisation remains controversial. Indeed rapid vasoconstrictive effects of anti-angiogenic drugs on tumour vessels have been reported that restrict rather than improve access to chemotherapy (Van der Veldt et al, 2012). Most likely, this highlights the importance of the judicious dosing of anti-VEGF therapy (i.e., doses high enough to reverse vessel abnormalities but not so high as to cause excessive vessel regression). Given that normalisation depends on achieving a balance between pro- and anti-angiogenic factors, this concept is supported by studies of sunitinib treatment of glioblastoma multiforme in which only low (but not high) doses of anti-VEGF treatment improved chemotherapy delivery into tumours (Kabbinavar, 2003; Zhou et al, 2008; Zhou and Gallo, 2009).
The key finding of the present study is, therefore, that compared with targeting VEGF or Ang2 individually, dual targeting in addition to reducing tumour growth and sprouting angiogenesis significantly boosts vascular normalisation parameters, including leakiness, hypoxia and perfusion as prerequisites for improved access for chemotherapy. Furthermore, we show for the first time that the formation of vascular BMS that facilitate the rapid vascular regrowth associated with resistance to VEGF-targeting drugs can be eliminated by dual targeting.

In agreement with previous reports, dual inhibition of VEGF and Ang2 induced synergistic inhibitory effects on tumour growth (Brown et al, 2010; Hashizume et al, 2010; Koh et al, 2010; Kienast et al, 2013) that were closely matched by the reduction in overall vessel density (Figure 1). Although Ang2 is typically associated with vascular destabilisation and remodelling, the observed reduction in vessel density is consistent with the demonstration of Ang2 having an additional role in sprouting angiogenesis during early tumour development (Nasarre et al, 2009; Felcht et al, 2012). During early stages of tumour development, inhibition of Ang2 prevents sprouting angiogenesis, by interfering with integrin signalling independently of its cognate receptor TIE2. During later stages, Ang2 inhibition promotes vascular normalisation by permitting the recruitment of stabilizing pericytes and improving the barrier function that leads to a reduction in interstitial fluid 
pressure. Reflecting these distinct functions, dual inhibition of Ang2 and VEGF leads to a further reduction in the tumour vessel density and a more mature vessel phenotype compared with bevacizumab alone. Our data clearly show that dual inhibition of Ang2 and VEGF provides complementary benefits associated with vascular normalisation and improved endothelial integrity including increased pericyte coverage, enhanced VE-cadherin tight junctions and reduced permeability (Figures 2 and 3 ).

Recent evidence indicates that sprouting angiogenesis may in principle be supported by both Ang2 and VEGF (Felcht et al, 2012). Accordingly, when either Ang2 or VEGF is effectively blocked leading to transient vessel regression and increasing hypoxia, there is a compensatory drive for sprouting angiogenesis that restores perfusion and prevents a further increase in tumour cell death. However, following effective dual inhibition of VEGF and Ang2 (L1/ $\mathrm{B} 100)$, the capacity for sprouting angiogenesis and therefore the ability to escape hypoxia by vascular remodelling should be lastingly impaired. As a result, the blood supply in hypoxic tumour areas may not be restored easily, explaining the increased tumour cell death observed in the dual targeting group (Figure 4A). Given that hypoxia and reoxygenation cycles are strong stimuli for neoangiogenesis that drive therapeutic resistance and metastasis (Dewhirst et al, 2008; De Bock et al, 2011), our finding of increased cell death at the expense of tumour hypoxia suggests that dual inhibition could offer a therapeutic strategy to reduce these risks. Equally, the increased stability and perfusion of the residual tumour vessels in the surviving and well-oxygenated tumour areas should not only benefit the penetration of cytostatic drugs, but also lower the frequency of metastatic dissemination (Holopainen et al, 2012), and create a more supportive tumour microenvironment for immuno- or chemotherapy (Huang et al, 2012).

When endothelial cells regress after VEGF inhibition, they leave empty vascular BMS that are durable and not degraded by macrophages and persist long after the blood vessels have disappeared (Inai et al, 2004). The accumulation of BMS thus provides a record of previously existing blood vessels (Inai et al, 2004) The second major finding of this study is that dual inhibition of Ang2 and VEGF prevents the formation of these type IV collagen containing BMS. We propose that the remarkable absence of BMS observed upon dual inhibition may indicate the more rapid closure of the vascular plasticity window, characterised by minimal neoangiogenesis and vascular remodelling. From a cancer therapeutic perspective BMS are a significant concern because they may provide a scaffold for vascular regrowth in tumours after cessation of treatment (Inai et al, 2004; Mancuso et al, 2006). In fact, previous attempts at preventing the formation of BMS using metalloproteinase inhibitors and the monoclonal antibody HUIV26 directed against cryptic domains of denatured type IV collagen were unsuccessful (Mancuso et al, 2006). On this basis, our demonstration that dual inhibition of Ang2 and VEGF can abolish the formation of BM deposits represents a novel strategy to significantly reduce this relapse risk.

Angiopoetin-2 is presently among the most intensely explored target molecules for the development of second-generation antiangiogenic drugs (Huang et al, 2010). Our findings suggest that dual inhibitors targeting both the Ang2 and VEGF pathways may provide complementary benefits, not only in terms of reducing tumour angiogenesis but perhaps even more importantly with regard to vascular normalisation. These findings are important, considering that such treatment options are close to entering the clinical arena. A Phase I clinical trial for a bi-functional CovXBody, CVX-241, which targets both Ang2 and VEGF, is ongoing. Recently, Ang-2-VEGF-A CrossMab, a novel bevacizumab-based bispecific human IgG1 antibody, was shown to combine potent anti-tumour, anti-angiogenic and anti-metastatic activity in preclinical testing supporting the investigation of the CrossMab in clinical trials (NCT01688206) (Kienast et al, 2013).
The present study argues that dual inhibition of VEGF and Ang2 in combination with cytostatic agents could provide a well justified and promising approach and should be explored further. Furthermore, our findings show that when Ang2 is inhibited tumour growth control and vascular normalisation can be achieved with significantly reduced doses of bevacizumab without incurring BMS that could foster resistance to VEGF targeting drugs. Further studies should clarify whether this benefit truly translates into to a more lasting response to anti-angiogenic therapy.

In summary, in this study we show for the first time that the dual targeting of Ang2 and VEGF provides complementary benefits in terms of vascular normalisation that cannot be achieved by inhibiting each pathway on its own. Inhibition of Ang2 specifically potentiates the normalizing activity of low-dose bevacizumab. Importantly, effective dual inhibition appears to be limiting the plasticity of the tumour vasculature by restricting the capacity of tumour vessels to induce neoangiogenesis in response to hypoxia and other pro-angiogenic stimuli associated with resistance to VEGF-targeting drugs. From a therapeutic perspective, this state of 'inert'-normalisation combining vascular normalisation with minimal plasticity could render tumours more sensitive to therapeutic intervention. In addition, the lack of BMS could counteract potential resistance mechanisms and prolong the effectiveness of VEGF-targeting drug. On this basis, further clinical studies should clarify whether VEGF-targeting drugs benefit from the concomitant inhibition of the Ang2 signalling axis, in terms of both effectiveness and duration of the clinical response.

\section{ACKNOWLEDGEMENTS}

We wish to acknowledge the kind support of Dr Jonathan D Oliner of Amgen Inc., who provided the Angiopoietin-2 blocking peptibody L1-10. HK and $\mathrm{MH}$ received funding from the Collaborative Research Centre SFB832.

\section{CONFLICT OF INTEREST}

The authors declare no conflict of interest.

\section{REFERENCES}

Batchelor TT, Duda DG, di Tomaso E, Ancukiewicz M, Plotkin SR, Gerstner E, Eichler AF, Drappatz J, Hochberg FH, Benner T, Louis DN, Cohen KS, Chea H, Exarhopoulos A, Loeffler JS, Moses MA, Ivy P, Sorensen AG, Wen PY, Jain RK (2010) Phase II study of cediranib, an oral pan-vascular endothelial growth factor receptor tyrosine kinase inhibitor, in patients with recurrent glioblastoma. J Clin Oncol 28: 2817-2823.

Bergers G, Hanahan D (2008) Modes of resistance to anti-angiogenic therapy. Nat Rev Cancer 8: 592-603.

Brown JL, Cao ZA, Pinzon-Ortiz M, Kendrew J, Reimer C, Wen S, Zhou JQ, Tabrizi M, Emery S, McDermott B, Pablo L, McCoon P, Bedian V, Blakey DC (2010) A human monoclonal anti-ANG2 antibody leads to broad antitumor activity in combination with VEGF inhibitors and chemotherapy agents in preclinical models. Mol Cancer Ther 9: 145-156.

Chae SS, Kamoun WS, Farrar CT, Kirkpatrick ND, Niemeyer E, de Graaf AM, Sorensen AG, Munn LL, Jain RK, Fukumura D (2010) Angiopoietin-2 interferes with anti-VEGFR2-induced vessel normalization and survival benefit in mice bearing gliomas. Clin Cancer Res 16: 3618-3627.

Dang DT, Chun SY, Burkitt K, Abe M, Chen S, Havre P, Mabjeesh NJ, Heath EI, Vogelzang NJ, Cruz-Correa M, Blayney DW, Ensminger St WD, Croix B, Dang NH, Dang LH (2008) Hypoxia-inducible factor-1 target genes as indicators of tumor vessel response to vascular endothelial growth factor inhibition. Cancer Res 68: 1872-1880.

De Bock K, Mazzone M, Carmeliet P (2011) Antiangiogenic therapy, hypoxia, and metastasis: risky liaisons, or not? Nat Rev Clin Oncol 8: 393-404. 
Dewhirst MW, Cao Y, Moeller B (2008) Cycling hypoxia and free radicals regulate angiogenesis and radiotherapy response. Nat Rev Cancer 8: $425-437$.

Ellis LM, Hicklin DJ (2008) VEGF-targeted therapy: mechanisms of anti-tumour activity. Nat Rev Cancer 8: 579-591.

Felcht M, Luck R, Schering A, Seidel P, Srivastava K, Hu J, Bartol A, Kienast Y, Vettel C, Loos EK, Kutschera S, Bartels S, Appak S, Besemfelder E, Terhardt D, Chavakis E, Wieland T, Klein C, Thomas M, Uemura A, Goerdt S, Augustin HG (2012) Angiopoietin-2 differentially regulates angiogenesis through TIE2 and integrin signaling. J Clin Invest 122: 1991-2005.

Fiedler U, Scharpfenecker M, Koidl S, Hegen A, Grunow V, Schmidt JM, Kriz W, Thurston G, Augustin HG (2004) The Tie-2 ligand Angiopoietin-2 is stored in and rapidly released upon stimulation from endothelial cell Weibel-Palade bodies. Blood 103: 4150-4156.

Gerald D, Chintharlapalli S, Augustin HG, Benjamin LE (2013) Angiopoietin-2: an attractive target for improved antiangiogenic tumor therapy. Cancer Res 73: 1649-1657.

Goede V, Coutelle O, Neuneier J, Reinacher-Schick A, Schnell R, Koslowsky TC, Weihrauch MR, Cremer B, Kashkar H, Odenthal M, Augustin HG, Schmiegel W, Hallek M, Hacker UT (2010) Identification of serum angiopoietin-2 as a biomarker for clinical outcome of colorectal cancer patients treated with bevacizumab-containing therapy. Br J Cancer 103: 1407-1414.

Hashizume H, Falcon BL, Kuroda T, Baluk P, Coxon A, Yu D, Bready JV, Oliner JD, McDonald DM (2010) Complementary actions of inhibitors of angiopoietin-2 and VEGF on tumor angiogenesis and growth. Cancer Res 70: $2213-2223$.

Holopainen T, Saharinen P, D'Amico G, Lampinen A, Eklund L, Sormunen R, Anisimov A, Zarkada G, Lohela M, Helotera H, Tammela T, Benjamin LE, Yla-Herttuala S, Leow CC, Koh GY, Alitalo K (2012) Effects of angiopoietin-2-blocking antibody on endothelial cell-cell junctions and lung metastasis. J Natl Cancer Inst 104: 461-475.

Huang H, Bhat A, Woodnutt G, Lappe R (2010) Targeting the ANGPT-TIE2 pathway in malignancy. Nat Rev Cancer 10: 575-585.

Huang Y, Yuan J, Righi E, Kamoun WS, Ancukiewicz M, Nezivar J, Santosuosso M, Martin JD, Martin MR, Vianello F, Leblanc P, Munn LL, Huang P, Duda DG, Fukumura D, Jain RK, Poznansky MC (2012) Vascular normalizing doses of antiangiogenic treatment reprogram the immunosuppressive tumor microenvironment and enhance immunotherapy. Proc Natl Acad Sci USA 109: 17561-17566.

Hurwitz H, Fehrenbacher L, Novotny W, Cartwright T, Hainsworth J, Heim W, Berlin J, Baron A, Griffing S, Holmgren E, Ferrara N, Fyfe G, Rogers B, Ross R, Kabbinavar F (2004) Bevacizumab plus irinotecan, fluorouracil, and leucovorin for metastatic colorectal cancer. N Engl J Med 350: $2335-2342$.

Inai T, Mancuso M, Hashizume H, Baffert F, Haskell A, Baluk P, Hu-Lowe DD, Shalinsky DR, Thurston G, Yancopoulos GD, McDonald DM (2004) Inhibition of vascular endothelial growth factor (VEGF) signaling in cancer causes loss of endothelial fenestrations, regression of tumor vessels, and appearance of basement membrane ghosts. Am J Pathol 165: 35-52.

Jain RK (2001) Normalizing tumor vasculature with anti-angiogenic therapy: a new paradigm for combination therapy. Nat Med 7: 987-989.

Jain RK, Duda DG, Clark JW, Loeffler JS (2006) Lessons from phase III clinical trials on anti-VEGF therapy for cancer. Nat Clin Pract Oncol 3: $24-40$.

Kabbinavar F (2003) Phase II, randomized trial comparing bevacizumab plus fluorouracil (FU)/leucovorin (LV) with FU/LV alone in patients with metastatic colorectal cancer. J Clin Oncol 21: 60-65.

Kienast Y, Klein C, Scheuer W, Raemsch R, Lorenzon E, Bernicke D, Herting F, Yu S, The HH, Martarello L, Gassner C, Stubenrauch KG, Munro K, Augustin HG, Thomas M (2013) Ang-2-VEGF-A CrossMab, a novel bispecific human IgG1 antibody blocking VEGF-A and Ang-2 functions simultaneously, mediates potent anti-tumor, anti-angiogenic, and antimetastatic efficacy. Clin Cancer Res 19: 6730-6740.

Koh YJ, Kim HZ, Hwang SI, Lee JE, Oh N, Jung K, Kim M, Kim KE, Kim H, Lim NK, Jeon CJ, Lee GM, Jeon BH, Nam DH, Sung HK, Nagy A, Yoo OJ, Koh GY (2010) Double antiangiogenic protein, DAAP, targeting VEGF-A and angiopoietins in tumor angiogenesis, metastasis, and vascular leakage. Cancer Cell 18: 171-184.

Lalande ME, Ling V, Miller RG (1981) Hoechst 33342 dye uptake as a probe of membrane permeability changes in mammalian cells. Proc Natl Acad Sci USA 78: 363-367.

Mancuso MR, Davis R, Norberg SM, O’Brien S, Sennino B, Nakahara T, Yao VJ, Inai T, Brooks P, Freimark B, Shalinsky DR, Hu-Lowe DD, McDonald DM (2006) Rapid vascular regrowth in tumors after reversal of VEGF inhibition. J Clin Invest 116: 2610-2621.

Mazzone M, Dettori D, Leite de Oliveira R, Loges S, Schmidt T, Jonckx B, Tian Y-M, Lanahan AA, Pollard P, Ruiz de Almodovar C, De Smet F, Vinckier S, Aragonés J, Debackere K, Luttun A, Wyns S, Jordan B, Pisacane A, Gallez B, Lampugnani MG, Dejana E, Simons M, Ratcliffe P, Maxwell P, Carmeliet P (2009) Heterozygous deficiency of PHD2 restores tumor oxygenation and inhibits metastasis via endothelial normalization. Cell 136: 839-851.

Nasarre P, Thomas M, Kruse K, Helfrich I, Wolter V, Deppermann C, Schadendorf D, Thurston G, Fiedler U, Augustin HG (2009) Host-derived angiopoietin-2 affects early stages of tumor development and vessel maturation but is dispensable for later stages of tumor growth. Cancer Res 69: 1324-1333.

Netti PA, Hamberg LM, Babich JW, Kierstead D, Graham W, Hunter GJ, Wolf GL, Fischman A, Boucher Y, Jain RK (1999) Enhancement of fluid filtration across tumor vessels: implication for delivery of macromolecules. Proc Natl Acad Sci USA 96: 3137-3142.

Ooyama A, Oka T, Zhao H, Yamamoto M, Akiyama S, Fukushima M (2008) Anti-angiogenic effect of 5-Fluorouracil-based drugs against human colon cancer xenograftsCancer Lett 267: 26-36.

Rolny C, Mazzone M, Tugues S, Laoui D, Johansson I, Coulon C, Squadrito ML, Segura I, Li X, Knevels E (2011) HRG inhibits tumor growth and metastasis by inducing macrophage polarization and vessel normalization through downregulation of PlGF. Cancer Cell 19: 31-44.

Sandler A, Gray R, Perry MC, Brahmer J, Schiller JH, Dowlati A, Lilenbaum R, Johnson DH (2006) Paclitaxel-carboplatin alone or with bevacizumab for non-small-cell lung cancer. N Engl J Med 355: 2542-2550.

Tong RT, Boucher Y, Kozin SV, Winkler F, Hicklin DJ, Jain RK (2004) Vascular normalization by vascular endothelial growth factor receptor 2 blockade induces a pressure gradient across the vasculature and improves drug penetration in tumors. Cancer Res 64(11): 3731-3736.

Van der Veldt AaM, Lubberink M, Bahce I, Walraven M, de Boer MP, Greuter HNJM, Hendrikse NH, Eriksson J, Windhorst AD, Postmus PE, Verheul HM, Serné EH, Lammertsma AA, Smit EF (2012) Rapid decrease in delivery of chemotherapy to tumors after anti-VEGF therapy: implications for scheduling of anti-angiogenic drugs. Cancer Cell 21: 82-91.

Zhou Q, Gallo JM (2009) Differential effect of sunitinib on the distribution of temozolomide in an orthotopic glioma model. Neuro Oncol 11: 301-310.

Zhou Q, Guo P, Gallo JM (2008) Impact of angiogenesis inhibition by sunitinib on tumor distribution of temozolomide. Clin Cancer Res 14: $1540-1549$.

This work is published under the standard license to publish agreement. After 12 months the work will become freely available and the license terms will switch to a Creative Commons AttributionNonCommercial-Share Alike 4.0 Unported License.

Supplementary Information accompanies this paper on British Journal of Cancer website (http://www.nature.com/bjc) 